\title{
Design of Stabilizing Controller for Networked Control Systems
}

\author{
Gaofeng Che ${ }^{1}$, Wei He${ }^{2}$, Tzong-Jer Chen ${ }^{3}$ \\ College of Information Engineering, Baise University, China \\ 1'chexuping@163.com; 2350977176@qq.com; 3d838502@alumni.nthu.edu.tw
}

Keywords: network-induced delay; LMI; delay-dependent; networked control systems

\begin{abstract}
This work is devoted to design the stabilizing state feedback controller for stability of the networked control systems. An improved stability criterion for networked control systems is proposed in the derivative of Lyapunov functions based on the relationship between the networked-induced delays and their bound. The stabilizing state feedback controller is applied to generate next control information for each subsystem using delayed sensing data in free-weighting LMI formulations. Moreover the design of controller of networked control systems focuses on the network-induced delays which may deteriorate the stability performance of networked control systems and even destabilize the real-time control of closed-loop systems. Simulation example shows the interest of the proposed approach.
\end{abstract}

\section{Introduction}

Although networked control systems(NCSs) have several advantages such as low installation cost, easy maintenance and so on. The use of communication networks makes it necessary to deal with network-induced delays. These delays may be unknown and time-varying, and may deteriorate the closed-loop systems ${ }^{[1-2]}$.

As for network-induced delays, they have been deal with by the control theory community in most research work. The performance and stability of control scheme strongly rely on the respect of the specified sampling rates and network-induced delays. Recently, the delay-dependent stabilizing method on the MADB has attracted much attention for the stability of NCSs with network-induced delays $^{[3]}$. A free-weighting matrix approach ${ }^{[4]}$ is reported to cover the results using Moon el al.'s inequality and the descriptor system approach ${ }^{[5]}$. As for NCSs, the delays are less than the sampling period for continuous-time systems based on sampling-rate method. Some methods to calculate the MADB for NCSs using Moon el al.'s inequality for both discrete-time plants are proposed in[6].

The stability analysis and controller design are dealt with in the presence of the network-induced delays. The object is to keep stability and good performance for NCSs in the presence of timing uncertainties as communication delays. Thus, it can be useful to consider more dynamic solutions.

\section{Design of NCSs Model and Stability Analysis}

We are interested here in real-time control of systems with communication delays (see Fig.1). There are mainly two sources of delays from the network-induced delays in an NCS: device delays and transmission delays. The computing delays include the time delay at the plant node (P) $\tau_{\text {sou }}$ and the controller node $(\mathrm{C}) \tau_{\text {des }}$. The transmission delays include the time delay from sensor node $(\mathrm{S})$ to controller node $(\mathrm{C}) \tau_{s c}$ and from controller node (C) to actuator node (A) $\tau_{c a}$. The total time delay can be expressed as follows

\subsection{NCSs model}

$$
\tau=\tau_{\text {sou }}+\tau_{\text {des }}+\tau_{s c}+\tau_{c a}
$$

In this section, we will review and improve some results on the modeling of NCSs. We need the following assumptions:

Assumption 1. In an NCS, the total time delay $\tau$ is less than one sampling period $h$. 
Assumption 2. In an NCS, the NCS uses the way of single-packet transmission. The data packet loss and noise effect on the NCS are not considered.

Assumption 3. In an NCS, the sensor is assumed to be time-driven, whereas the controller and actuator are event-driven, and controller is time-invariant.

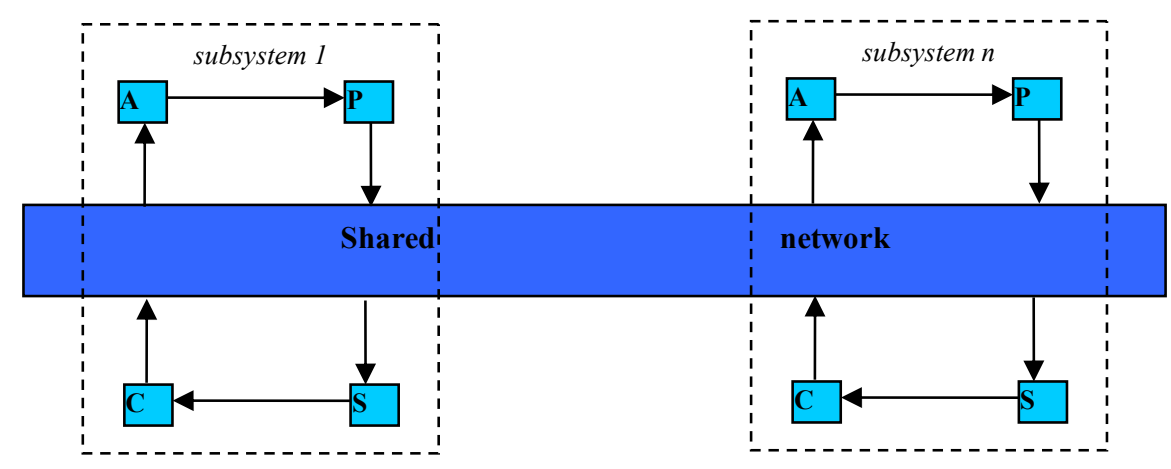

Figure 1. Networked Control Systems Structure

The discrete system equations can be written as:

$$
\begin{gathered}
x(k+1)=A_{\tau} x(k-\tau)+B u(k) \\
x(k)=0, \quad(-\tau \leq k \leq 0)
\end{gathered}
$$

where, state vector $x(k) \in \mathfrak{R}^{n}$; control input vector $u(k) \in \mathfrak{R}^{m} ; A, A_{\tau}$ and $B$ are real constant matrices with appropriate dimensions.

The total time delay $\tau$ satisfies:

$$
\tau_{1} \leq \tau \leq \tau_{2}
$$

where, $\tau_{1}$ and $\tau_{2}$ are minimum and maximum time delay respectively and less than one sampling time.

\subsection{Controller Design}

The memoryless state feedback controller for the NCSs is designed.

$$
u(k)=K x(k)
$$

where, $u(k)=0, k \in(-\tau, 0)$.

Lemma 4. (Schur complements) Given constant matrices $\mathrm{M}, \mathrm{P}$ and $\mathrm{Q}$, where $P=P^{T}$ and $Q=Q^{T}<0$, then, $P-M Q^{-1} M^{T}<0$, if only if $\left(\begin{array}{cc}P & M \\ M^{T} & Q\end{array}\right)<0$, or $\left(\begin{array}{ll}Q & M^{T} \\ M & P\end{array}\right)<0$.

Theorem 5. Given $\tau_{i}>0(i=1,2)$, if there exist constant matrices $P, Q, Z, X, N_{1}, N_{2}$, where $P=P^{T}>0, Q=Q^{T} \geq 0, Z=Z^{T} \geq 0, X=\left(\begin{array}{cc}X_{11} & X_{12} \\ * & X_{22}\end{array}\right) \geq 0, N_{1}$ and $N_{2}$ have appropriate dimensions, hold,

$$
\begin{gathered}
\Phi=\left(\begin{array}{ccc}
\Phi_{11} & \Phi_{12} & (A-I)^{T} H \\
* & \Phi_{22} & A_{\tau}^{T} H \\
* & * & H
\end{array}\right)<0 \\
\tilde{X}=\left(\begin{array}{ccc}
X_{11} & X_{12} & N_{1} \\
* & X_{22} & N_{2} \\
* & * & Z
\end{array}\right) \geq 0
\end{gathered}
$$

then, the $\operatorname{system}(2)$ with the time-delay constraint(4) is stable when $u(k)=0$. 
where, $\Phi_{11}=\left(\tau_{2}-\tau_{1}+1\right) Q+P(A-I)+\left(A-I^{T}\right) P+N_{1}+N_{1}^{T}+\tau_{2} X_{11}, H=P+\tau_{2} Z, \Phi_{12}=P A_{\tau}+N_{2}^{T}-N_{1}+\tau_{2} X_{22}$, $\Phi_{22}=-Q-N_{2}-N_{2}^{T}+\tau_{2} X_{22}$.

Proof. Given $y(k)=x(k+1)-x(k)$, then $x(k+1)=x(k)+y(k)$.So,

$$
x(k)-x(k-\tau)-\sum_{k=1}^{n} y(l)=0
$$

Choose Lyapunov functional candidates as: $\quad V(k)=V_{1}(k)+V_{2}(k)+V_{3}(k)$, $V_{1}(k)=x^{T}(k) P x(k), V_{2}(k)=\sum_{\theta=-\tau_{2}+1}^{0} \sum_{l=k-1+\theta}^{k-1}\left(y^{T}(l) Z y(l)\right), V_{3}(k)=\sum_{\theta=-\tau_{2}+1}^{-\tau_{1}+1} \sum_{l=k-1+\theta}^{k-1}\left(x^{T}(l) Q x(l)\right)$, where, $P=P^{T}>0, Q=Q^{T} \geq 0$ and $Z=Z^{T} \geq 0$.

Let $\Delta V(k)=V(k+1)-V(k)$, then

$\Delta V_{1}(k)=x^{T}(k+1) P x(k+1)-x^{T}(k) P x(k)=2 x^{T}(k) P y(k)+y^{T}(k) P y(k)$

$\Delta V_{2}(k)=\tau_{2} y^{T}(k) Z y(k)-\sum_{l=k-\tau}^{k-1}\left(y^{T}(l) Z y(l)\right) \leq \tau_{2} y^{T}(k) Z y(k)-\sum_{l=k-\tau}^{k-1}\left(y^{T}(l) Z y(l)\right)$

$\Delta V_{3}(k)=\left(\tau_{2}-\tau_{1}+1\right) x^{T}(k) Q x(k)-\sum_{l=k-\tau_{2}}^{k-\tau_{1}}\left(x^{T}(l) Q x(l)\right) \leq\left(\tau_{2}-\tau_{1}+1\right) x^{T}(k) Q x(k)-x^{T}(k-\tau) Q x(k-\tau)$

The differential function of Lyapunov function $V(k)$ is written as follows:

$$
\begin{aligned}
\Delta V(k) & =2 x^{T}(k) P y(k)+y^{T}(k) P y(k)+\tau_{2} y^{T}(k) Z y(k)-\sum_{l=k-\tau}^{k-1}\left(y^{T}(l) Z y(l)\right)+\left(\tau_{2}-\tau_{1}+1\right) x^{T}(k) Q x(k) \\
& -x^{T}(k-\tau) Q x(k-\tau)
\end{aligned}
$$

Equation (8) and matrices $N_{1}(i=1,2)$ are used, the written equation is as follows:

$$
2\left[x^{T}(k) N_{1}+x^{T}(k-\tau) N_{2}\right] \times\left[x(k)-x(k-\tau)-\sum_{l=k-\tau}^{k-1} y(l)\right]=0
$$

On the other hand, as for any matrix $X=\left(\begin{array}{cc}X_{11} & X_{12} \\ * & X_{22}\end{array}\right) \geq 0$, the following equation holds:

$$
\sum_{l=k-\tau_{2}}^{k-1}\left(\zeta_{1}^{T}(l) X \zeta^{T}(l)\right)-\sum_{l=k-\tau}^{k-1}\left(\left(\zeta_{1}^{T}(l) X \zeta^{T}(l)\right)\right)=\tau_{2} \zeta_{1}^{T}(l) X \zeta^{T}(l)-\sum_{l=k-\tau}^{k-1}\left(\left(\zeta_{1}^{T}(l) X \zeta^{T}(l)\right)\right)
$$

where, $\zeta_{1}(k)=\left[\begin{array}{ll}x^{T}(k) & x^{T}(k-\tau)\end{array}\right]$, add the left sides of equation(9) and equation(10) into $\Delta V(k)$, hence $\quad$ we $\quad \Delta V(k) \leq \zeta_{1}^{T}(k) \Xi \zeta_{1}(k)-\sum_{l=k-\tau}^{k-1}\left(\zeta_{2}^{T}(k) \Psi \zeta_{2}(k)\right)$ $\zeta_{2}(k, l)=\left[\begin{array}{ll}\zeta_{1}^{T}(k) & y^{T}(l)\end{array}\right]^{T}, \Xi=\left(\begin{array}{ll}\Phi_{11}+(A-I)^{T} H(A-I) & \Phi_{12}+(A-I) H A_{\tau} \\ \Phi_{12}^{T}+A_{\tau}^{T} H(A-I)^{T} & \Phi_{22}+A_{\tau}^{T} H A_{\tau}\end{array}\right)$.

So, an NCS is stable is only if $\Xi<0$ and $\Psi>0$. According to Lemma 4 , we know that $\Xi<0$ is equal to $\Phi<0$.

Theorem 6. Given $\tau_{i}>0(i=1,2)$, if there exist constant matrices $L, W, R, Y, M_{1}, M_{2}, V$, they satisfy that $L=L^{T}>0, W=W^{T} \geq 0, R=R^{T} \geq 0, Y=\left(\begin{array}{ll}Y_{11} & Y_{12} \\ * & Y_{22}\end{array}\right) \geq 0, M_{1}, M_{2}$ and $V$ have appropriate dimensions. So, 


$$
\begin{aligned}
\Pi & =\left(\begin{array}{llll}
\pi_{11} & \pi_{12} & \pi_{12} & \tau_{2} \pi_{11} \\
* & \pi_{22} & \pi_{23} & \tau_{2} L A_{\tau}^{T} \\
* & * & -L & 0 \\
* & * & * & -\tau_{2} R
\end{array}\right)<0 \\
\tilde{Y} & =\left(\begin{array}{lll}
Y_{11} & Y_{12} & M_{1} \\
* & Y_{22} & M_{2} \\
* & * & L R^{-1} L
\end{array}\right) \geq 0
\end{aligned}
$$

then, the system(2)with the time-delay constraint(4) can be controlled and $K=V L^{-1}$, $\pi_{11}=\left(\tau_{2}-\tau_{1}+1\right) W+A L+L A^{T}-2 L+B V+V^{T} B^{T}+M_{1}+M_{1}^{T}+\tau_{2} Y_{22} \quad, \quad \pi_{12}=A_{\tau} L+M_{2}^{T}-M_{1}+\tau_{2} Y_{12}$, $\pi_{13}=L(A-I)^{T}+V^{T} B^{T}$, and $\pi_{22}=-W-M_{2}-M_{2}^{T}+\tau_{2} Y_{22}$.

Proof. According to the proof of Theorem 5, the LMI (6) is changed by using Lemma 4.

$$
\bar{\Phi}=\left(\begin{array}{lllc}
\Phi_{11} & \Phi_{12} & (A-I)^{T} P & \tau_{2}(A-I) Z \\
* & \Phi_{22} & A_{\tau}^{T} P & \tau_{2} A_{\tau}^{T} Z \\
* & * & -L & 0 \\
* & * & * & -\tau_{2} Z
\end{array}\right)<0
$$

then, the $\operatorname{NCSs}(8)$ under the control of the memoryless state feedback controller and its state equation is

$$
x(k+1)=(A+B K) x(k)+A_{\tau} x(k-\tau)
$$

As for the system (14), $A+B K$ is used to instead of $A$ in the LMI(13), then the LMI(13) is

$$
\tilde{\Phi}=\left(\begin{array}{cccc}
\tilde{\Phi}_{11} & \tilde{\Phi}_{12} & (A+B K-I)^{T} P & \tau_{2}(A+B K-I) Z \\
* & \tilde{\Phi}_{22} & A_{\tau}^{T} P & \tau_{2} A_{\tau}^{T} Z \\
* & * & -L & 0 \\
* & * & * & -\tau_{2} Z
\end{array}\right)<0
$$

where, $\quad \tilde{\Phi}_{11}=\left(-\tau_{2}-\tau_{1}+1\right) Q+P(A+B K-I)+(A+B K-I)^{T} P+N_{1}+N_{1}^{T}+\tau_{2} X_{11} \quad, \quad \tilde{\Phi}_{12}=P A_{\tau}+N_{2}-N_{2}^{T}+\tau_{2} X_{12}$, $\tilde{\Phi}_{22}=-Q-N_{2}-N_{2}^{T}+\tau_{2} X_{22}$.

So, the method of proof for Theorem 5 is used, then

$$
\begin{array}{r}
\operatorname{diag}\left\{P^{-1}, P^{-1}, P^{-1}, Z^{-1}\right\} \bullet \tilde{\Phi} \bullet \operatorname{diag}\left\{P^{-1}, P^{-1}, P^{-1}, Z^{-1}\right\}<0 \\
\quad \operatorname{diag}\left\{P^{-1}, P^{-1}, P^{-1}\right\} \bullet \tilde{X} \bullet \operatorname{diag}\left\{P^{-1}, P^{-1}, P^{-1}\right\} \geq 0
\end{array}
$$

where, $Y=\operatorname{diag}\left\{P^{-1}, P^{-1}\right\} \bullet X \bullet \operatorname{diag}\left\{P^{-1}, P^{-1}\right\} \quad, \quad L=P^{-1}, W=P^{-1} Q P^{-1} \quad, R=Z^{-1}, M_{1}=P^{-1} N_{1} P^{-1}$, $M_{2}=P^{-1} N_{2} P^{-1}, V=K P^{-1}$.

The final conclusion is drawn by calculating the $\operatorname{LMIs}(16),(17)$.

\section{Simulation}

In this section, one example is given to show effectiveness of the proposed method. It is solved by the LMIs from Theorem 5 and Theorem 6.

According to the system state equation (2), when $u(k)=0$, the system matrices are denoted as follows:

$$
A=\left(\begin{array}{ll}
0.8 & 0 \\
0 & 0.97
\end{array}\right), \quad A_{\tau}=\left(\begin{array}{ll}
-0.1 & 0 \\
-0.1 & -0.1
\end{array}\right)
$$

where, $\tau_{1}=\tau_{2}, \tau$ is constant. 
If the methods in [7],[8] is used, the maximum values of $\tau_{2}$ are 12 and 16 respectively. however, its values is 16 used the method proposed in this paper.

As for network-induced delay $\tau_{1}$, the methods in [7],[8] and in this paper are used respectively. The results as follows

Table 1. Upper Bound of $\tau$

\begin{tabular}{|c|c|c|c|c|c|c|}
\hline$\tau_{1}$ & 1 & 3 & 5 & 7 & 9 & 11 \\
\hline Fridman\&Sharked & 8 & 8 & 8 & 8 & 8 & 8 \\
\hline Theorem5,6 & 10 & 10 & 10 & 10 & 11 & 12 \\
\hline
\end{tabular}

From TABLE 1, we know that the results uses the method in this paper is less conservative than that uses the method of Fridman and Sharked

\section{Conclusions}

The stability of NCSs is analysed through an improved stability criteria that proposed in the derivative of Lyapunov function and consider the relationship between the network-induced delays which are time-varying. A stabilizing state feedback controller is applied in each subsystem of NCSs in a free-weighting LMIs formulation. Simulation example shows the effectiveness of the method.

\section{Acknowledgments}

This work is financially supported by Project Foundation (Project Number:2014KB06) granted by Baise University and Project Foundation (Project Number:KY2015LX388) granted by Department of Education of Guangxi Zhuang Autonomous Region of China.

\section{References}

[1] K.Gu, V.L.Kharitonov, J.Chen, Stability of time-delay systems, 2003.

[2] G.Che,Y.Jin,Online co-design of feedback control and real-time scheduling for embedded systems with communication delays, ICCSE 2009,pp.618-623,2009.

[3] E.Fridman,U.Shaked,Delay-dependent stability and H1 control :constant and time-varying delays, Int. J. Control, pp.48-60,2003.

[4] Y.He,M.Wu,J.H.She,G.P.Liu,Delay-dependent robust stability criteria for uncertain neural systems with mixed delays, Syst.Control Lett.,pp.57-65,2004.

[5] Y.He, M.Wu, J.H.She, G.P.Liu,Paremeter-dependent Lyapunov functional for stability of time-delay systems with polytopic-type uncertainties, IEEE Trans.Autom. Control, pp.828-832,2004.

[6] H.Park,Y.Kim,D.Kim,W.H.Kwon,A scheduling method for network based control systems,IEEE Control Syst.Tech.,pp.318-330,2002.

[7] Y.S.Lee, W.H.Kwon, Delay-dependent robust stabilization of uncertain discrete-time state-delayed systems, Proceeding of 15th IFAC Congress Automation and Control of Barcelona,2002.

[8] E.Fridman,U.Sharked, An LMI approach to stability of discrete delay systems,EuropeanControl Conference, 2003. 